Apidologie, 1974, 5 (3) 225-246.

\title{
NEUE UNTERSUCHUNGEN ZUM « DOPPELTEN UMLARVEN »
}

\author{
Nouvelles recherches sur le double transfert des larves
}

Karl WEISS

Bayer. Landesanstalt für Bienenzucht, Erlangen

\section{SUMMARY}

NEW INVESTIGATIONS OF ( DOUBLE GRAFTING )

Queen cells built over " double grafted " larvae - i.e. larvae which had been transferred on the remaining broodfood in queen cell cups in which previously larvae had been bred for 24 hours and then removed - were drawn out longer than those built over "single grafted" or "dry grafted" larvae. Until pupation, there was more royal jelly hoarded in the queen cells containing double grafted larvae than in those with single grafted ones, and after pupation a larger amount of food remainders was left.

When larvae, aged 24 hours, were transferred on the brood food of larvae grafted at the same age and removed after a 24 hours breeding time at an age of almost 2 days the queens did not weigh more than those reared from single grafted larvae. Sometimes they even weighed less. When larvae, 1 day old, were transferred to the brood food of larvae of their age the relations seemed to be reverse, though the differences in weight were not quite convineing. The presumable causes of this phenomenon are discussed.

Only after transferring larvae aged $11 / 2$ and 2 days on the brood food of larvae being their age or even older ones, the developing pupae were heavier than those reared from dry grafted larvae. The characteristies typical for queens, as number of ovarioles, shape of head, mandibles and tarsi did not vary. - The attempt is made to explain the different influences of single and double grafting on the development of larvae of various ages presuming that older larvae, with a greater food consumption, are more sensitive than younger ones against a food deficiency possibly occurring after single grafting.

Compared to single grafting, double grafting of larvae aged 24 hours or less is of no advantage for practical queen rearing. 


\section{ZUSAMMENFASSUNG}

Über “ doppelt umgelarvten » Maden — das sind Maden, die in künstliche Weiselbecher auf den Futtersaft von 24 Stunden angebrüteten und dann entfernten Erstlarven übertragen wurden - entstanden längere Weiselzellen als über c einfach » und « trocken 》 umgelarvten Tieren. In der Zelle mit “ doppelt " umgelarvten Maden sammelte sich bis zur Verpuppung mehr Futtersaft an, und die unverbrauchten Restmengen nach der Verpuppung waren größer als beim « einfachen » Umlarven.

Als knapp 1-tägige Maden auf den Futtersaft von 24, Stunden angebrüteten und beim Entfernen knapp 2 Tage alten Erstlarven gelegt wurden, waren die daraus entstandenen Königinnen nicht schwerer als die aus einfach umgelarvten Vergleichsmaden gezogenen - in Einzelzuchten sogar statistisch gesichert leichter. Legte man 1-tägige Maden auf Futtersaft gleichaltriger Larven, so schienen sich die Verhältnisse umzukehren, wenn auch die Gewichtsunterschiede wenig überzeugend waren. Die vermutliche Ursache hierfür wird erörtert.

Erst als $11 / 2$ und 2 Tage alte Larven auf Futtersaft von gleichaltrigen oder älteren Tieren übertragen wurden, entwickelten sich vorwiegend schwerere Puppen als aus trocken umgebetteten Vergleichsmaden. Hinsichtlich der königinnentypischen Merkmale : Ovariolenzahl, Form des Kopfes, der Kiefer und der Hinterbeinfersen bestand jedoch kein Unterschied. - Der unterschiedliche Einfluß des einfachen und doppelten Umlarvens auf das Wachstum verschieden alter Larven wird damit zu erklären versucht, daß ältere Maden bei stärkerem Futterverbrauch gegenüber einer möglichen, nach dem einfachen Umlarven auftretenden kurzfristigen Ernährungslücke empfindlicher reagieren als jüngere Tiere.

Für die praktische Zuchtarbeit erbringt das “ doppelte Umlarven » bei Verwendung von 1-tägigen oder jüngeren Maden gegenüber der einfachen Umbettung keinerlei Vorteile.

Die U̇bertragung junger Arbeiterlarven in vorgeformte Weiselbecher aus Wachs oder Kunststoff ist die meistgeübte Methode der Vorbereitung des Zuchtstoffes für die Aufnahme ins Pflegevolk. Dabei legt man die Arbeitermade entweder in das trockene Näpfchen oder man versieht dieses unmittelbar vorher mit einem Tropfen Königinnenfuttersaft. Der Futtersaft wird in der Regel aus angebrüteten Schwarmzellen gesammelt; man verwendet ihn naturbelassen, oder mit etwas Wasser verdünnt, notfalls auch nach kurzer Aufbewahrungszeit im Kühlschrank. Anstelle dieses sog. " feuchten » Umlarvens wendet man vielfach auch die Methode des « doppelten » Umlarvens an. Dabei überträgt man in die trockenen Weiselbehälter zunächst junge Maden beliebiger Abstammung, entfernt diese nach kurzer Anbrütezeit (meist nach 24 Stunden) und bettet auf den inzwischen abgelagerten Futtersaft die endgültigen Zuchtmaden. Beide Verfahren sind in Züchterkreisen weit verbreitet, teils weil die mit Futter vorversorgten Näpfchen eine bessere Annahme ergeben sollen als die trocken belarvten, teils weil man mit Vorteilen bei der Larvenentwicklung und besserer Ausbildung der Zuchtprodukte rechnet.

Das Umlarven auf Weiselfuttersaft hat geschichtliche Wurzeln. So ist der Amerikaner G. M. Doolitwle, der 1889 in seinem Lande das Umlarven in künstliche Weiselbecher in allen Einzelheiten publik machte, mit trockenen Näpfchen überhaupt nicht zurecht gekommen. In Deutschland hat der Vater des modernen Umlarvverfahrens, W. W. WANkLER, in den 90iger Jahren 
des vergangenen Jahrhunderts ebenfalls von Anfang an Futtersaft zur Übertragung der Larven in die von ihm bevorzugten natürlichen Näpfchen oder Drohnenzellen benützt (“ Die Königin ), 1. u. 2. Auflage 1903). In jüngeren vergleichenden Untersuchungen ist einmal von Annahmevorteilen (VuiLLAume 1959, Montagner 1962, Örösi Pál 1966), einmal von indifferentem Verhalten der Bienen gegenüber mit Futtersaft versorgten Näpfchen die Rede (FrEE u. Spencer-Booth 1961). Böttcher u. Weiss fanden 1962, daß in starken, in voller Pflegekraft stehenden weisellosen Völkern zwischen trocken umgebetteten Maden und solchen, die auf eine kleine Menge frischen, aus Weiselwiegen mit etwa 2 Tage alten Maden entnommenen Futtersaft gelegt wurden, kein nennenswerter Unterschied in der Annahme bestand, während schwächere Pfleger Larven auf Futtersaft zu bevorzugen schienen.

Ungeachtet der offenen Diskussion über Annahmevorteile der feucht bzw. doppelt umgelarvten Maden herrscht in der Bienenliteratur über die viel wichtigere Frage, ob sich die auf Futtersaft umgetteten Maden besser entwickeln und “ bessere 》 Königinnen ergeben als trocken umgelarvte, keine einheitliche Meinung. Die Frage ist bisher hauptsächlich in Verbindung mit dem doppelten Umlarven untersucht worden, welches man gewöhnlich als eine Art Vervollkommnung des feuchten Umlarvens ansieht. Vuillaume (1959) berichtet von einer besseren Weiterverpflegung der doppelt umgelarvten Maden und weist auf die Möglichkeit hin, größere Königinnen mit größerer Fruchtbarkeit damit zu erzielen. WEAver (1957) hat außer einer (ohnehin schwer meßbaren! Verf.) größeren Samenblase der doppelt umgelarvten Königinnen keine weiteren Unterschiede z.B. in der Abdomenlänge, dem Durchmesser und der Ovariolenzahl der Eierstöcke und in der Länge der Mandibeldrüsen gegenüber einfach umgelarvten Königinnen gefunden. In einem Versuch Montagners (1962) entwickelten sich die doppelt umgelarvten Maden zułgrößeren Königinnen mit größerer Ovariolenzahl als die einfach umgebetteten, wobei jedoch keine Korrelation zwischen Schlupfgewicht und Ovariolenzahl bestand (nur 6 Königinnen in jeder Gruppe!). Während ÖrösI PÁL 1952 und 1960 darauf hinwies, daß der in einen Weiselbecher übertragene Futtersaft bei fehlendem Nachschub oft schon nach 1030 Minuten eingetrocknet sein kann, wogegen die umgebetteten Maden oft erst nach Stunden weitergep flegt werden, schlof er 1963 aus Versuschen, bei denen er den Futtersaft der Pflegevölker durch Verfütterung von gefärbter Zuckerlösung farbig markierte, daß die Versorgung der Maden beim doppelten Umlarven (je nach dem Zustand des Volkes!) schneller und sicherer vor sich gehe, als bei einmaliger Umbettung auf einen Futtersafttropfen. WoHLGemutH (1933) glaubte ein etwas höheres und einheitlicheres Schlupfgewicht doppelt umgelarvter Königinnen im Vergleich zu einfach, aber auf einen Tropfen Futtersaft umgebetteten Tieren festgestellt zu haben. 13 doppelt umgelarvte Königinnen wogen 1/2-1 $1 / 2$ Tage (!) nach dem Schlüpfen im Durchschnitt 
$220 \mathrm{mg}$ und waren um durchschnittlich $7 \mathrm{mg}$ schwerer als 27 Vergleichstiere (aus einem anderen Pflegevolk!). Der geringe Unterschied war zudem bei der Nachwägung der begatteten Tiere verschwunden. Nach einem Bericht von H. MaUl hat Wolosiewitsch 1954 nach 2 maligem Umlarven Königinnen gezogen, die in Größe und Ausbildung der Geschlechtsorgane den auf einen Honigtropfen einfach umgelarvten Tieren überlegen waren. Auch Mǎrza 1965 hält auf Grund von Wägungen das doppelte Umlarven für vorteilhaft. Auf der anderen Seite gibt JoRDAN 1956 zu bedenken, daß der Königinnenfuttersaft, welchen die zweitumgelarvte Made im Weiselbehälter vorfindet, altersmäßig — und er meint damit auch qualitativ — nicht zu ihr passe.

Wie die experimentellen Untersuchungen über den Wert des « feuchten » und “ doppelten ) Umlarvens in der Durchführung und den Ergebnissen zumindest unbefriedigend sind, so ist auch in der Praxis die Meinung über die Zweckmäßigkeit dieser Maßnahmen geteilt. Um nur einige Beispiele zu nennen, kennt das renommierte Zuchtbuch “ Queen Rearing » von Snelgrove, 3. Auflage 1966, ausschließlich das feuchte Umlarven, wobei « double grafting 》 besonders herausgestellt wird. Jüngere Artikel in amerikanischen Bienenzeitungen über die Königinnenproduktion pochen geradezu auf dieses Verfahren (Hastings 1967, Farrar 1968). Dagegen rücken Laidlaw und ECKERT in ihrem Buch « Queen Rearing ) 1962 vom doppelten Umlarven ab, da sie es für möglich halten, daß die Bienen den Futtersaft ausräumen und von neuem mit der Fütterung beginnen. Sie halten aber weiter an der Vorversorgung der Näpfchen mit Futtersaft fest, vorausgesetzt, daß nicht mit einem Spezialumlarvapparat Futtersaft gleichzeitig mit der Larve aufgenommen und übertragen werden kann. ZANDER emp fiehlt in seinem Handbuch der Bienenkunde Band V « Die Zucht der Biene », bis in die 1953 erschienene 8. Auflage das Umlarven auf eine Futtersaftunterlage, wenngleich er von Pechaczex (1928) weiß, daß es auch ohne Futtersaft geht. Dagegen will er vom doppelten Umlarven nicht viel wissen. Erst die 9. von Böтtcher überarbeitete Auflage von 1971 rückt vom feuchten Umlarven ab, wogegen dem doppelten Umlarven wiederum mehr Chancen eingeräumt zu werden scheinen.

Die unbestimmte Situation in der Frage der Versorgung der Weiselnäpfchen mit Futtersaft beim Umlarven fordert zur Neuaufnahme von vergleichenden Versuchen geradezu heraus. Die theoretisch beste Form der Verproviantierung der Weiselwiegen ist das doppelte Umlarven. In der folgenden Untersuchung geht es um die alte Frage, ob und wieweit das doppelte Umlarven den Wert der entstehenden Königinnen zu beein flussen vermag. Das Gewicht und, als Ergänzung, die Ausbildung einiger wichtiger kastentypischer Merlmake bilden dabei die Bewertungsgrundlage. 


\section{I. - GEWINNUNG DES UNTERSUCHUNGSMATERIALS UND METHODIK}

Die Königinnen für die vergleichende Untersuchung wurden in Pflegevölkern gezüchtet, deren Stockmutter 9 Tage vor Zuchtbeginn in den Honigraum abgesperrt war. Zwei Stunden vor Zuchtbeginn wurde sie mitsamt der offenen Brut entfernt. In den so vorbereiteten Völkern züchtete ich in wöchentlichem Abstand meist zu wiederholten Malen. Für jeden Zuchtansatz verwendete ich einen Zuchtrahmen mit 32 oder 48 Weiselbechern - zuerst aus Wachs, später aus Plastik. Die Becher versah ich mit bestimmtaltrigen Maden. Nach 24 Stunden larvte ich zum zweiten Mal um. Ich hätte einfach die Hälfte der angenommenen Maden entfernen und durch eine neue Madengarnitur ersetzen können, wobei die andere Hälfte der vortags umgelarvten Maden als Vergleich gedient hätte. Dieses Verfahren, welches gelegentlich in Versuchen von anderen Autoren angewendet wurde, hat nicht nur den Nachteil, da $\beta$ die zu vergleichenden Maden verschiedenen Legephasen der jeweiligen Zuchtkönigin entstammen, sie erscheinen auch zu verschiedenen Zeiten im Pflegevolk, während sich dessen Annahme- und Pflegebereitschaft bereits geändert haben kann. Ich habe deshalb stets sämtliche angebrüteten Erstlarven entfernt. Gleichzeitig ersetzte ich die Hälfte der angep flegten Näpfchen durch leere Weiselbecher und belarvte sie zusammen mit den verbleibenden angep flegten Bechern mit gleichaltrigen Geschwisterlarven. Trockene und vorversorgte Näpfchen wurden in alternierender Reihenfolge neu geordnet.

Die zu den Versuchen verwendeten Larven hatten genau datiertes Alter. Sie wurden durch Absperren der Königin für 6 bis höchstens 12 Stunden auf eine leere Wabe unter einem Aufsteckkäfig aus Absperrgitter gewonnen. In der Regel suchte ich die größten Maden zur Zucht aus, so da $\beta$ sich der Altersspielraum nach der oberen Altersgrenze hin noch einengte.

Die Zuchtrahmen kamen frühestens 5 Tage nach der Zweitbelarvung, also gleich nach dem Deckeln der zweiten Larvengarnitur in den Brutschrank. Aus den im Brutschrank heranreifenden Zellen entfernte ich die Königinnenpuppen 2 Tage vor dem errechneten Schlupftermin. Ich wog sie, legte sie auf den Boden hölzerner Schlupfkäfige, die mit Futterteig und Wasser ausgestattet waren und brachte sie in den Brutschrank zurück, wo sie sich ohne schützende Wachshülle weiter entwickelten (WEIss 1967). Etwa einen Tag nach Abschluß der Imaginalhäutung kamen die Königinnen bis zu ihrer weiteren Verarbeitung in $70 \%$ igen Alkohol. Später habe ich einen Teil der zu vergleichenden Tiere auf die Beschaffenheit ihrer kastentypischen Merkmale untersucht (s. dazu WeIss 1971). Bei der Tabellisierung der in allen Einzelheiten erfaßten Merkmale des Ovars, der Kopf- und Oberkieferform sowie der Beschaffenheit der Hinterbeine beschränkte ich mich jedoch auf die Wiedergabe einiger besonders markanter Werte. So wurde die Ausbildung der Eierstöcke nur in der Zahl der Ovariolen berücksichtigt, welche ich durch direktes Auszählen ermittelte. Zur Charakterisierung des Kopfes genügte der Gesichtsindex, in dem die Summe aus den kürzesten Entfernungen zwischen den beiden Facettenaugen im Wangen- $\left(b_{w}\right)$ und Stirnbereich $\left(b_{s t}\right)$ als Divident und die mediane Strecke zwischen dem unteren Rand des mittleren Oocellus und der ventralen Randbegrenzung des Kopfschildes (1) als Divisor auftritt. Die kastentypische Gestalt der Mandibel wurde durch das Verhältnis seiner größten Länge zur schmalsten Breite erfaßt und zur Kennzeichnung der Ferse diente als besonders empfindliches Maß der Quotient aus ihrer Länge und ihrer größten Breite. Soweit die zum Vergleich herangezogenen Merkmale bilateral ausgebildet sind, wurden sie in der rechnerischen Auswertung stets zusammen verarbeitet.

Nach dem Herausschneiden der Königinnenpuppen aus den Zellen, zwei Tage vor dem Schlupf, habe ich den in den Zellen befindlichen Futtersaftrest mit einem Spatel entnommen und sofort gewogen. Infolge seiner unterschiedlichen Konsistenz treten vorhandene Mengenunterschiede im Gewichtsvergleich besonders deutlich in Erscheinung.

Die hier ausgewerteten Zuchtversuche wurden über mehrere Jahre an der Bayerischen Landesanstalt für Bienenzucht in Erlangen und ergänzend 1972 an der Entomologischen Abteilung der Universität von Georgia in Athens, U.S.A. durchgeführt. Für die Unterstüttzung, die mir dort vom Vorstand der Bienenabteilung, Prof. A. Dietz, und dem Leiter des Departements, Prof. H. Lund, zuteil wurde, darf ich den Genannten freundlich danken. 


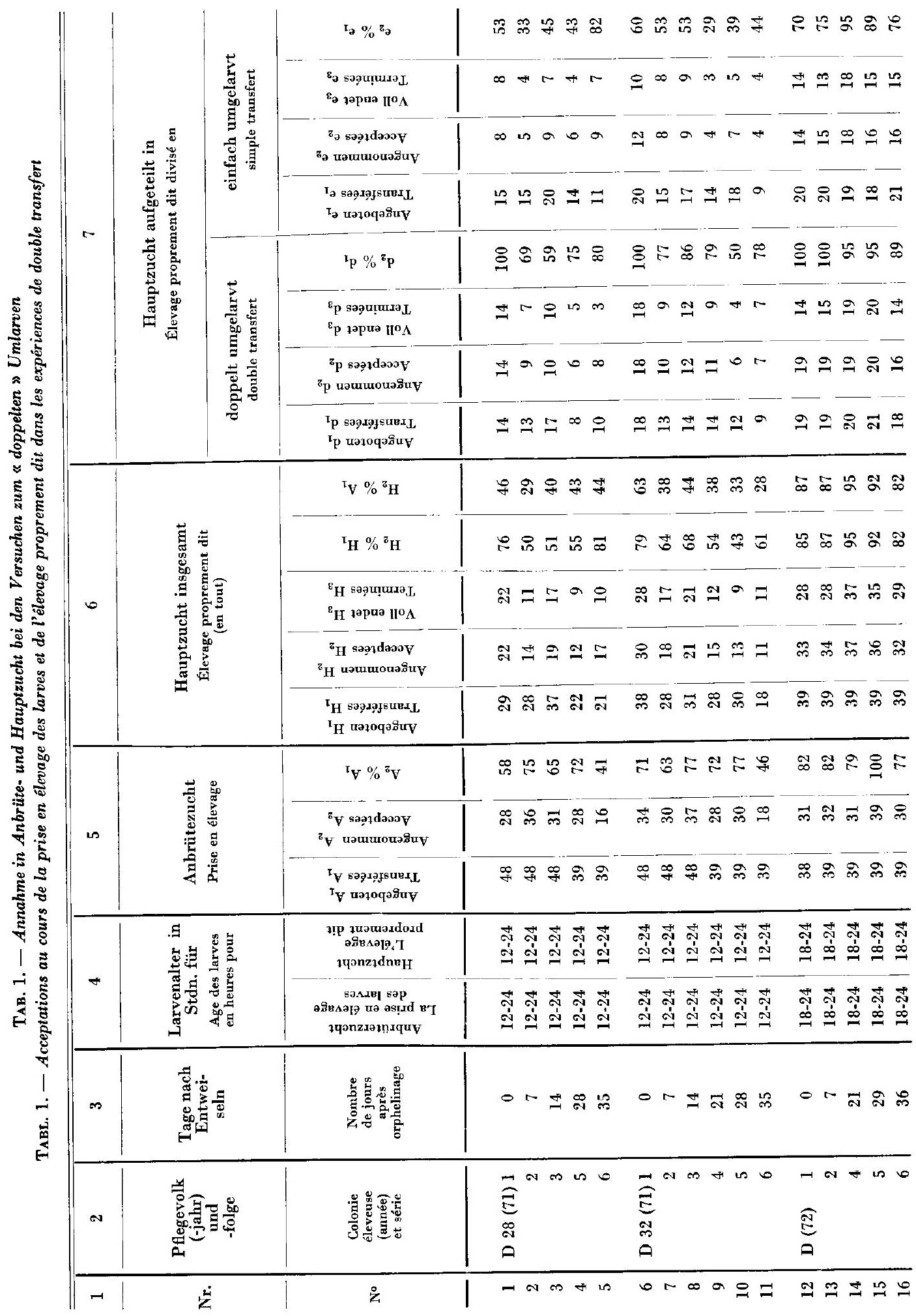




\section{II. - ERGEBNISSE DER VERGLEICHSZUCHTEN}

\section{a) Die Annahme}

Mit der Stärke der Pflegevölker, ihrem Zustand und ihrer Veranlagung wechselt die Bereitschaft zur Zellenp flege. Leider erlaubt die Art der Durchführung meiner Versuche keine präzise Aussage darüber, ob das doppelte Umlarven in der Praxis Annahmevorteile mit sich bringt. Trotzdem will ich in einer Generalübersicht über alle in dieser Untersuchung durchgeführten Zuchten versuchen, die Annahmeverhältnisse zu analysieren. In Tabelle 1 gibt jede Zeile über eine Zuchtserie Auskunft. Sie umfaßt Angaben über Pflegevolk, Pflegefolge, Alter der zur Erst- und Zweitzucht verwendeten Larven und, in den Spalten 5-7, die wesentlichen Zahlen über Larvenangebot und- annahme in der Anbrüte- und Hauptzucht mit prozentualen Vergleichen.

Wenn aus dem Larvenangebot der Anbrütezucht (Spalte 5, $\mathbf{A}_{1}$ ) alle Näpfchen als angenommen gelten, die nach einem Tag Anbrütezeit mit Futtersaft versorgte Maden enthielten $\left(A_{2}\right)$, muß man dem Larvenangebot bei der Hauptzucht (Spalte 6, $\mathrm{H}_{1}$ ) ebenfalls alle zu Anfang in Pflege genommenen Näpfchen $\left(H_{2}\right)$ gegenüberstellen und nicht etwa nur die am Ende aus der Zucht hervorgehende Zahl unversehrter Königinnen $\left(\mathrm{H}_{3}\right)$. Bei den Ausfällen, um die $\mathbf{H}_{3}$ kleiner ist als $\mathbf{H}_{2}$, handelt es sich $u$. a. um nur teilweise ausgezogene leere Weiselkrüge, Zellen mit abgerutschten Maden oder um gedeckelte Zellen, deren Inhalt als Streckmade, Vorpuppe oder Puppe abgestorben ist. Diese Ausfälle sind von Volk zu Volk und von Zucht zu Zucht verschieden - besonders groß mitunter auf späterer Zuchtfolge, wenn auch die Annahme bereits zu wünschen übrig läßt. In Zucht Nr. 31 ist ein großer Teil der Königinnen durch unbeabsichtigte Erschütterung des Zuchtrahmens gleich nach dem Deckeln der Zellen zugrunde gegangen.

Bei einem Vergleich der prozentualen Annahme in der Anbrütezucht (Spalte 5, $\mathbf{A}_{2} \% A_{1}$ ) mit der in der Hauptzucht (Spalte 6, $\mathbf{H}_{2} \% \mathbf{H}_{1}$ ) muß man berücksichtigen, daß bei der Hauptzucht meistens weniger Larven gegeben wurden als bei der Anbrütezucht, weil schon der Erstansatz zu wünschen übrig ließ. Dadurch errechnen sich bei der Hauptzucht nicht selten beträchtlich höhere Annahmewerte. Alle Versuche zusammengefaßt steht einem Annahmeprozentsatz von $66 \%$ in der Anbrütezucht ein solcher von $80 \%$ in der Hauptzucht gegenüber. Vergleicht man gerechter-, wenn auch statistisch nicht gerechtfertigterweise die Annahme in der Hauptzucht mit dem Larvenangebot in der Vorauszucht, so verschwindet dieser Unterschied, und es läßt sich sogar eine (unbedeutend) geringere Annahme in der Hauptzucht gegenüber 
der Anbrütezucht konstatieren (60\% gegen $66 \%$ ). Da in der Anbrütezucht nur trocken umgelarvt wurde, bei der Hauptzucht aber stets ein gewi iser Anteil doppelt umgelarvter Maden beteiligt war, würde das bedeuten, daß das doppelte Umlarven, insgesamt gesehen, die Annahme nicht verbessert hat.

Der Vergleich der prozentualen Annahmezahlen der doppelt und einfach umgelarvten Maden in der Hauptzucht (Spalte 7, $d_{2} \% d_{1}$ und $e_{2} \% e_{1}$ ) fällt in fast allen Einzelzuchten (bei vorhandener Auswahlmöglichkeit!) deutlich zu Gunsten der doppelt belarvten Zellen aus. Alle Versuche zusammen, beträgt die Annahme bei den doppelt umgebetteten Maden $89 \%$, bei den trocken umgelarvten $72 \%$. Um genügend Vergleichstiere aus einfach umgebetteten Maden zu bekommen, habe ich deshalb in der Hauptzucht oft von vornherein mehr Larven trocken als doppelt umgebettet (Vergl. Spalte 7, $d_{1}$ und $\left.e_{1}\right)$. Aber nicht alle Pflegevölker besitzen für die vorgepflegten Näpfchen eine gleichgroße Vorliebe. Drei starke Ligustica-Völker (D, ND, DN) die ich 1972 in Amerika zu Versuchen verwendete, schienen weniger wählerisch zu sein (Vers.Nr.12-21). Die gleichfalls starken Carnica-Pflegevölker F und Fb der Versuche von 1967 (Nr. 25-32) und DEa von 1973 (Nr. 34) wiesen so gut wie keinen Annahmeunterschied zwischen den beiden Umlarvarten auf. Verschiedene Völker scheinen sich hier individuell verschieden zu verhalten. $\mathrm{DaB}$ auch die allgemeine Pflegebereitschaft dabei eine Rolle spielt, mag man daraus ersehen, daß die Annahmeunterschiede zwischen doppelt und einfach umgelarvten Maden vornehmlich dann verhältnismäßig klein zu sein scheinen, wenn die Annahme insgesamt in einer Zuchtserie besonders gat ausfiel.

\section{b) Restfuttermengen in den Weiselzellen}

Meist schon beim Herausnehmen des gedeckelten Zuchtrahmens mit den wechselweise doppelt und einfach umgelarvten Maden fiel ein deutlicher und systematischer Größenunterschied der Zellen ins Auge. Die zweimal belarvten Zellen waren länger (Abb. 1). Was ist die Ursache?

Einen Anhaltspunkt liefert die Untersuchung der nach dem Verpuppen der Königinnen in der Weiselzelle zurückbleibenden Futtersaftrestmenge. Die Königinnenmade schwinmt während ihrer ganzen Entwicklungszeit in einem Futterüber fluß. Sie verbraucht nur einen Teil der ihr laufend dargereichten Nahrung. Nach dem Schlüpfen findet sich in der Regel ein mehr oder weniger großer Futtersaftrest in der Zelle. Der Umfang dieses Restfutters hängt weitgehend vom physiologischen Zustand und Alter des Pflegevolkes und von der Seriengröße und Anzahl der angenommenen Zellen ab. Er wird aber auch von der Darbietungsart des Zuchtstoffs in Form des “ einfachen » und « doppelten » Umlarvens beein flußt. 
Zur Demonstration dieses Sachverhaltes seien aus der Masse der Versuche nur 2 Beispiele herausgegriffen. Ich habe in Tabelle 2 den Zuchtgang in zwei Pflegevölkern mit jeweils mehreren aufeinanderfolgenden Zuchtserien dargestellt. Neben den orientierenden Angaben über Zuchtaufbau und -verlauf in den Spalten 2-7 sind in den Ergebnisspalten 8 und 9 die wichtigsten Zahlen aus der statistischen Bearbeitung der Restfuttergewichte in den Zellen mit doppelt und einfach umgelarvten Königinnen festgehalten.

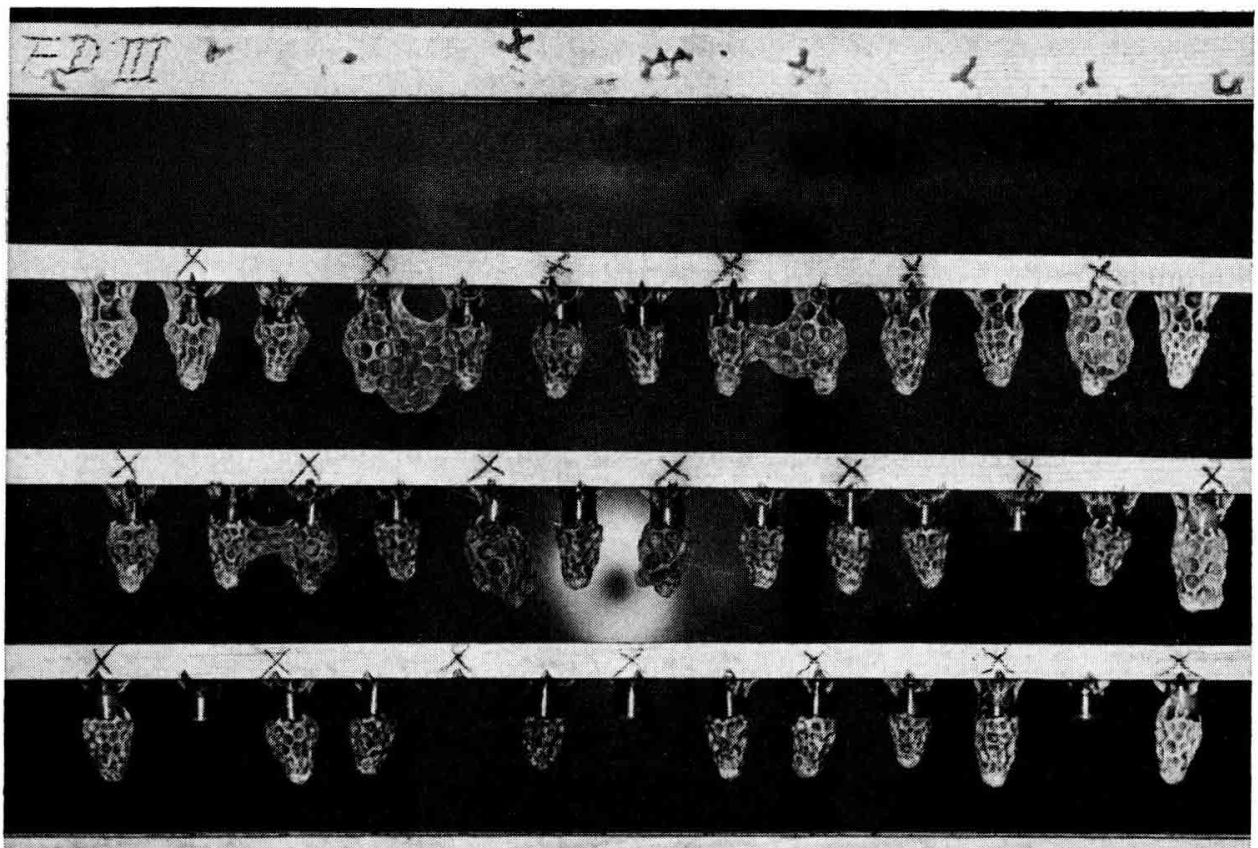

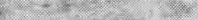

ABB. 1. Z Zuchtrahmen mit « einfach 》 und " doppelt " belarvten Zellen, aus Pflegefolge FD (72) 3. Zuchtserie. Die doppelt belarvten Zellen sind auf den Zuchtrahmen mit $x$ gekennzeichnet.

Fic. 1. - Cadre d'élevage avec cellules contenant des larves de simple et double transfert. Colonie éleveuse FD (72). $3^{\mathrm{e}}$ série. Les cellules contenant des larves provenant du double transfert sont marquées d'une croix.

Ein Blick auf die Gewichtsdifferenzen in Spalte 9 zeigt sofort ein starkes Überwiegen der Futtersaftrestmengen in den Zellen mit doppelt umgelarvten Tieren. Die Anfangsgversorgung der doppelt umgebetteten Zuchtlarven tritt also auch noch im unverbrauchten Futtersaftrest am Ende der Larvenentwicklung deutlich in Erscheinung. 


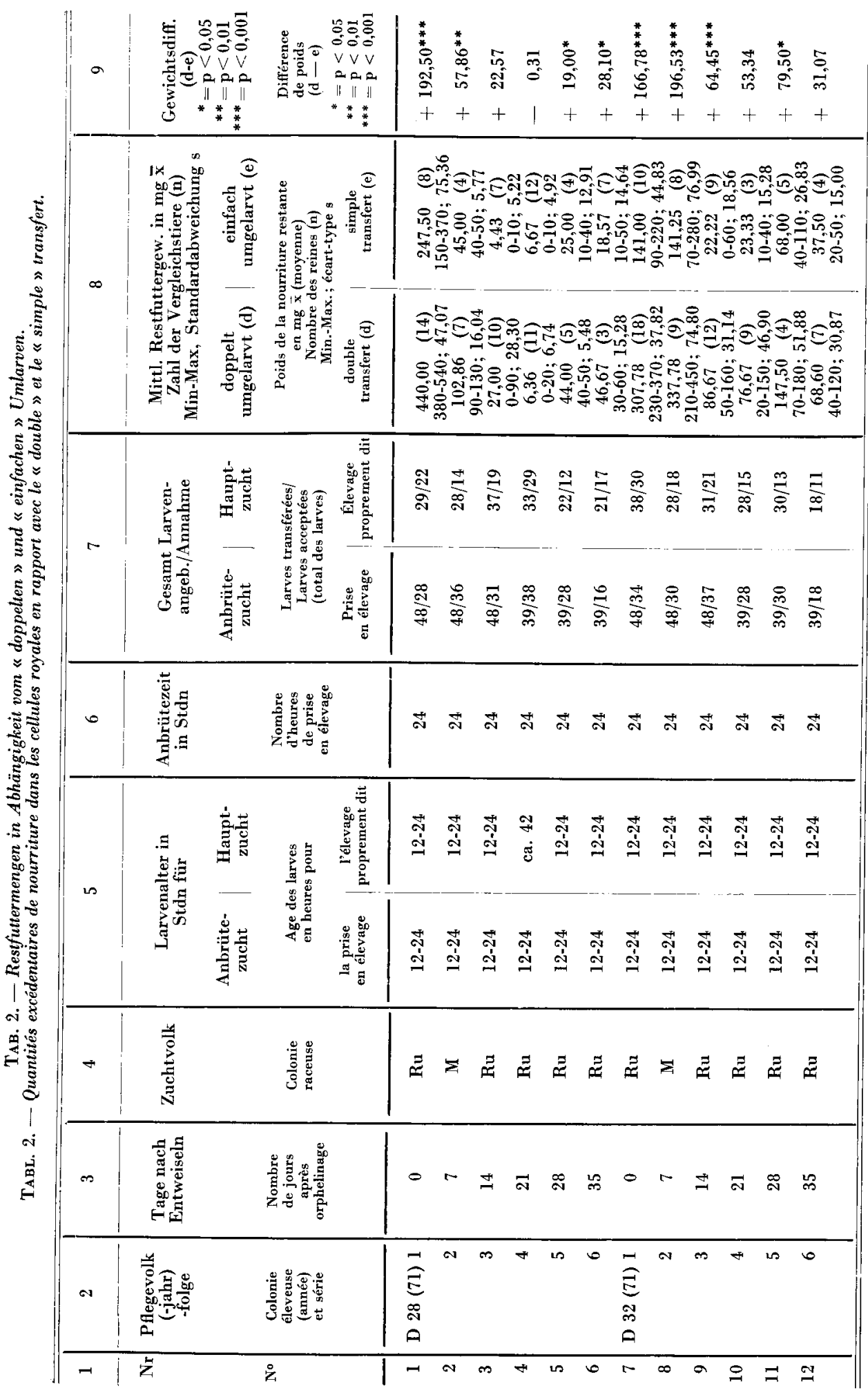


Allgemein nehmen die Restfuttersaftmengen sowohl der doppelt als auch der einfach umgelarvten Königinnen im Verlauf der Wiederholungszuchten rasch ab. Gelegentliche Unregelmäßigkeiten hierbei sind nur teilweise mit der Anzahl der in Pflege genommenen Zellen verknüpft, sonst aber schwer erklärbar. Daß mit Abnahme der Futtersaftrestgewichte im Verlauf der einzelnen Pflegefolgen auch die anfangs hohe Sicherung der Gewichtsdifferenzen nachläßt, ist bei den übrigbleibenden verhältnismäßig geringen Futtersaftresten nicht verwunderlich.

Bezeichnenderweise verlor sich mit dem Rückgang der Restfuttermengen und deren Gewichtsdifferenzen auch der zuerst besonders auffällige Unterschied in der Länge der Weiselzellen von doppelt und einfach umgelarvten Königinnen. Wenn die Zellen auch nicht ausgemessen, sondern nur nach dem Augenschein beurteilt wurden, so sorgte doch das Nebeneinander der beiden Zellenarten am gleichen Zuchtrahmen für eine ausreichende Sicherheit der Feststellung: Futtersaftrestmenge und die Weiselzellenlänge stehen in direkter Abhängigkeit zueinander.

\section{c) Königinnengewichte}

Man könnte vermuten, daß in den längeren Zellen mit den größeren Futtersaftresten doppelt umgebetteter Maden auch größere Königinnen entstünden. Gewichtsbestimmungen von Königinnenpuppen aus vergleichenden Zuchtversuchen mit doppeltem und einfachem Umlarven mögen darüber Aufschluß geben. Tabelle 3 umfaßt sämtliche zur Klärung dieser Frage durchgeführten Versuche. Sie entspricht in der Anlage Tabelle 2, nur sind in den Spalten 8 und 9 an Stelle der Restfuttermengen die Gewichte der Königinnenpuppen statistisch bearbeitet.

In der ersten Versuchsgruppe (Vers. Nr. 1-16) verwendete ich zum ersten und zum zweiten Umlarven 1/2- bis 1-tägige Maden. Die doppelt umgebetteten Larven kamen also auf den Futtersaft von inzwischen $11 / 2-2$ Tage alt gewordenen Maden zu liegen. In den 16 Zuchtansätzen waren einmal die einfach, einmal die doppelt umgelarvten Königinnen schwerer. In 3 Fällen ließ sich die Gawichtsdifferenz statistisch gerade noch bis gut absichern überraschenderweise wogen dabei jedesmal die Puppen der einfach umgelarvten Königinnen mehr. Bei der Zusammenfassung aller hier genannten Zuchten ergab die statistische Bearbeitung der Königinnengewichte mithilfe des parameterfreien Testverfahrens von Mann und Withney keinen echten Unterschied zwischen den einfach und doppelt umgelarvten Tieren.

In den beschriebenen Versuchen kamen die doppelt umgelarvten Zuchtmaden auf Futtersaft, der weder altersmäßig noch in der (zu reichlichen) Menge zu ihnen paßte. In den Zuchtansätzen der zweiten Versuchsgruppe Nr. 17-24 wurde dieser « Nachteil » durch Übertragung entsprechend jüngerer 
Maden beim ersten Umlarven behoben. Bei der Zweitbelarvung bettete ich ca. 1-tägige Maden auf den Futtersaft gleichaltriger Tiere. Diesmal häufen sich in Spalte 9 die Pluszeichen vor den Differenzwerten, was auf einen Gewichtsvorteil der Königinnen aus doppelt umgelarvten Maden hindeutet. Allerdings ist der errechnete durchschnittliche Gewichtsunterschied bei Zusammenfassung aller Zuchten mit $3,65 \mathrm{mg}$ gegenüber dem gewogenen Gewichtsmittel sämtlicher beteiligter Königinnenpuppen von $263,32 \mathrm{mg}$ außerordentlich gering. Nur einmal, in Vers. Nr. 18 ist die Gewichtsdifferenz zu Gunsten der doppelt umgelarvten Königinnen schwach gesichert. Alle Zuchtansätze zusammengefaßt, errechnet sich nach Mann und Withney eine Irrtumswahrscheinlichkeit von $\mathbf{p}=0,014$.

Die Zuchten Nr. 25-32 in der dritten Versuchsgruppe wurden mit älteren Maden in der Hauptzucht durchgeführt. Zur Erstbelarvung verwendete ich etwa 1/2 Tage alte Tiere. Nach 24. Stunden legte ich 1 1/2-tägige Larven auf den Futtersaft der entfernten, inzwischen gleichalten Erstlarven und in die trockenen Kontrollnäpfchen. Hier zeigte sich zum ersten Mal, daß doppelt umgelarvte Königinnen trocken umgebetteten Tieren gewichtsmäßig deutlich überlegen sein können. Der Unterschied ließ sich schon häufig in den Einzelzuchten statistisch schwach bis gut absichern.

In einer vierten Versuchsgruppe (Vers. Nr. 33-35) verwendete ich noch ältere Maden von knapp 2 Tagen zur Hauptzucht und benützte zur Erstbeschickung der Näpfchen, 24. Stunden vorher, einmal um ebensoviel jüngere und zweimal gleichaltrige Maden. In den beiden letzten Fällen kamen die doppelt umgebetteten Larven auf Futtersaft von knapp 3-tägigen, also um 1 Tag älteren Maden, zu liegen. Trotzdem waren in allen 3 Versuchen die Königinnen aus doppelt umgelarvten Maden denen aus einfach umgelarvten gewichtsmäßig deutlich überlegen. Die letzte nicht so überzeugende Zuchtserie Nr. 35 krankt an zu geringer Annahme der trocken umgebetteten Maden.

Die meisten der beschriebenen Zuchtversuche wurden als Wiederholungszuchten innerhalb längerer Pflegefolgen durchgeführt. In diesem Zusammenhang mag man fragen, ob das Pflegealter der Ammenbienen einen Einflu $\beta$ auf das Zuchtergebnis ausüben kann. Man könnte sich vorstellen, daß bei abnehmender Pflegekraft, bzw. geringer werdenden Futtersaftreserven der Völker die Vorteile einer Vorversorgung der doppelt umgelarvten Maden stärker zum Tragen kämen und in höheren Gewichtszunahmen zu Buche schlügen als zu Beginn der Sukzessivzuchten. Ein Vergleich der aufeinanderfolgenden Zuchtserien in der Differenzspalte der Tabelle 3 läßt erkennen, daß das nicht zutrifft. 


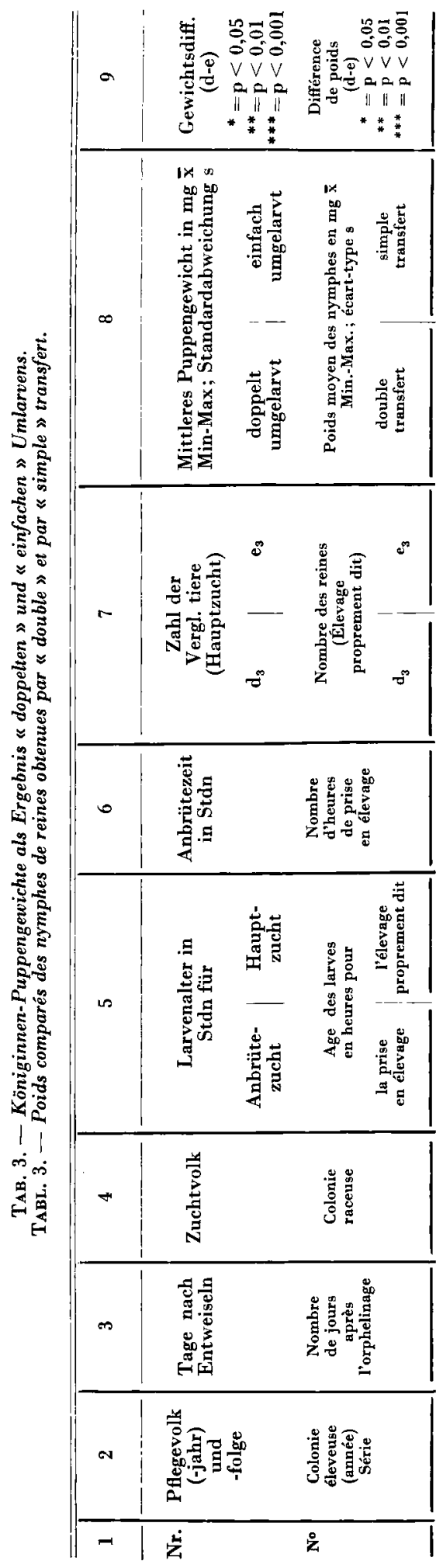

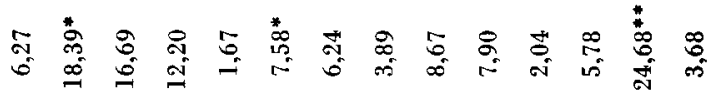

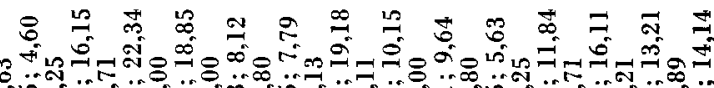

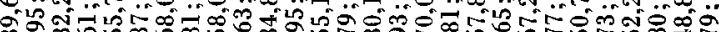

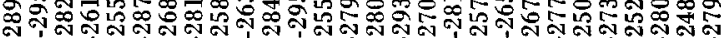

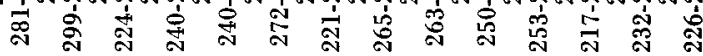

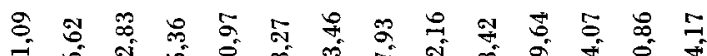
ஜ

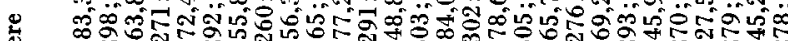

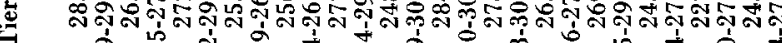
ن 悹点

茫空

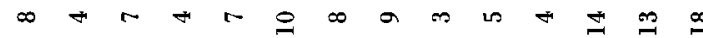

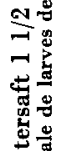

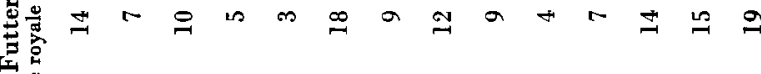
峞峞

害旨

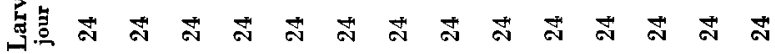

$\frac{\pi 000}{80 \pi}$

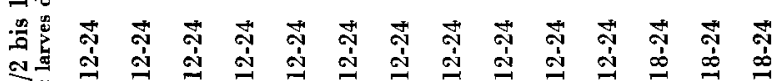
…

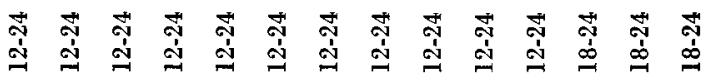

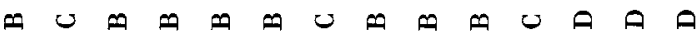

-

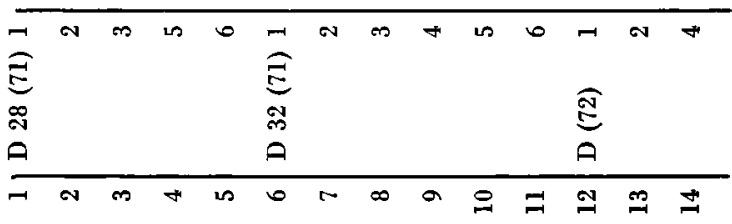


ڤ.

$++$

$12 \%$

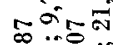

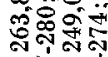
㲾

\section{ㅎ․ $8 \stackrel{\text { की }}{2}$

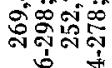 离 恋}

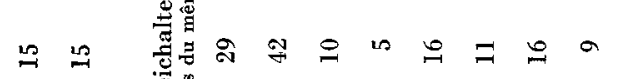

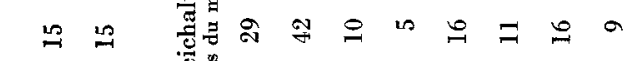

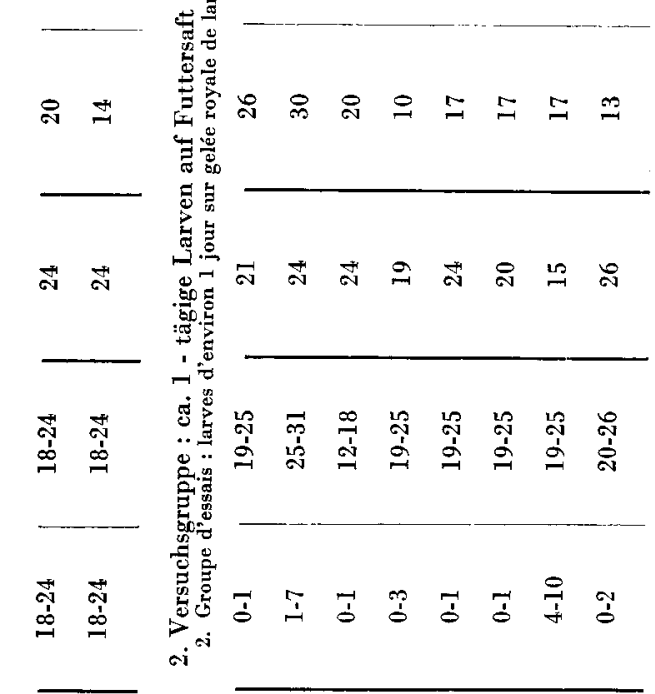

도

ลิ

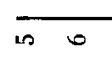

$\stackrel{2}{19}$ $5=\approx$ 。

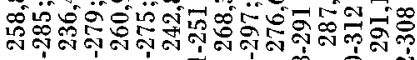
类

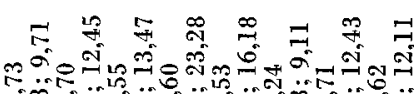
สึ้

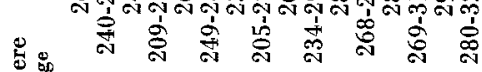

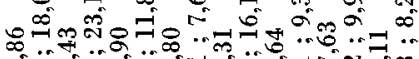

范

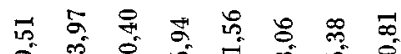
مึ๊

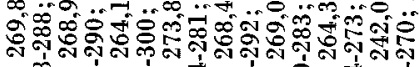

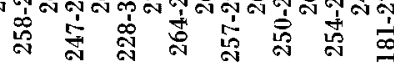
\%

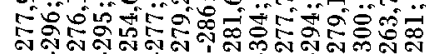
矛
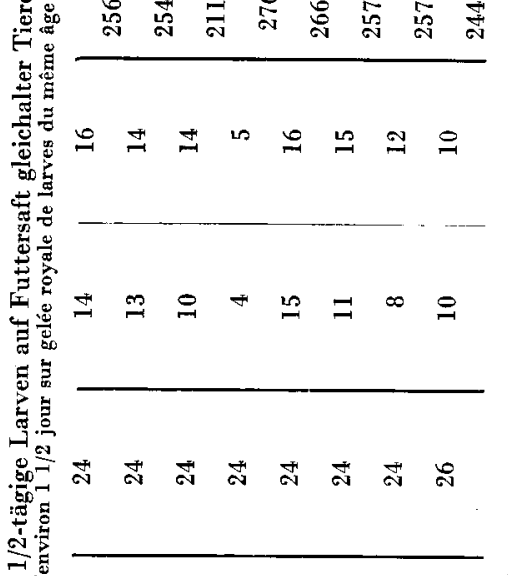

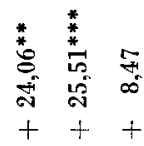

\& 8

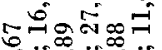

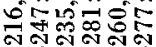
+口

迷

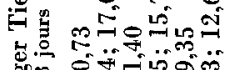
.500 옹 泟 N ․․․ 象 可

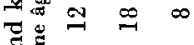

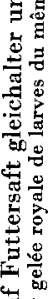
岂状芯 需盟 这 昰 $\therefore$

过

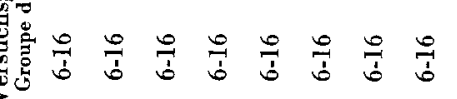
कं

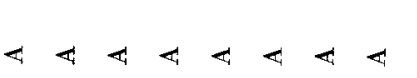

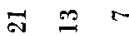

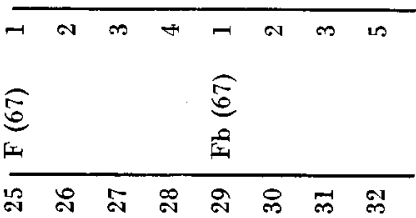

\begin{tabular}{|c|c|c|}
\hline 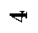 & is & $N$ \\
\hline$\overparen{F}$ & $\widehat{3}$ & $\widehat{3}$ \\
\hline$\infty$ & $=$ & 0 \\
\hline$\stackrel{N}{\rho}$ & 동 & 武 \\
\hline m & לת & 10 \\
\hline
\end{tabular}




\section{d) Ovariolenzahl und äußere Kastenmerkmale}

Die dem Gewicht nach unterschiedlichen Königinnen aus einfach und doppelt umgelarvten 1 1/2-tägigen Maden der dritten Zuchtgruppe Nr. 25-32 untersuchte ich schließlich noch auf den Grad ihrer kastentypischen Ausbildung. Wenn es Unterschiede zwischen diesen Tieren geben sollte, derart, daß die eine Gruppe einem fiktiven Vollweibchen näher käme als die andere, müßte das mit Hilfe so eindringlicher kastentypischer Kriterien wie Ovariolenzahl, Kopfform, Oberkiefer- und Hinterbeinausbildung am ersten nachzuweisen sein (s. Methodik). Tabelle 4 enthält die Ergebnisse dieser Merkmalsuntersuchungen für jede der fraglichen Zuchtserien. Die kleinen und vorzeichenverschiedenen Differenzwerte der durchschnittlichen Zähl- und Meßergebnisse zwischen den Königinnen aus doppelt und einfach umgelarvten Maden in den Spalten 5, 7, 9 und 11 sind ein Ausdruck für die Zufälligkeit der gegenseitigen Abweichungen. Niemals war ein Merkmalsunterschied innerhalb einer Zuchtserie statistisch zu sichern, ebenso wie die Zusammenschau aller Versuche nirgends auf einen echten Unterschied hinwies.

\section{DISKUSSION}

Die Ergebnisse der Versuche zum einfachen und doppelten Umlarven erscheinen im Hinblick auf die erreichten Puppengewichte zunächst komplex. Während die auf Futtersaft älterer Maden abgestreiften 1/2 bis 1-tägigen Larven keine größeren, in Einzelfällen sogar gesichert kleinere Tiere ergaben als die trocken umgelarvten, und während aus 1-tägigen, auf Futtersaft gleichaltriger Maden umgebetteten Larven so gut wie gleichschwere Tiere entstanden, entwickelten sich aus 1 1/2-tägigen und älteren Larven, gleich ob sie auf altersgleichen oder älteren Futtersaft gelegt wurden, deutlich schwerere Puppen als bei der einfachen Belarvung.

Um das mitunter geringere Wachstum der doppelt umgebetteten Tiere in der ersten Versuchsgruppe (Tab. 3) zu erklären, wird man zuerst an die Altersdiskrepanz zwischen den jungen Zuchtlarven und der Futtersaftunterlage denken. Wenn man dabei die Qualität des altersmäßig nicht genau zu den Larven passenden Futters im Auge hat, ist dieses Argument indessen wenig überzeugend. In früher durchgeführten Versuchen ergab der Vergleich von Königinnenpuppen, welche aus (trocken umgelarvten) verschieden alten bis knapp 2-tägigen Maden erbrütet wurden, einen nur mit Hilfe zahlreicher Versuche mühsam zu sichernden Gewichtsunterschied (Werss 1974). Wenn die Vorversorgung der Maden mit dem chemisch immerhin nachweislich vom Weiselfutter verschiedenen Arbeiterfuttersaft das Adultgewicht der 
DOPPELTES UMLARVEN

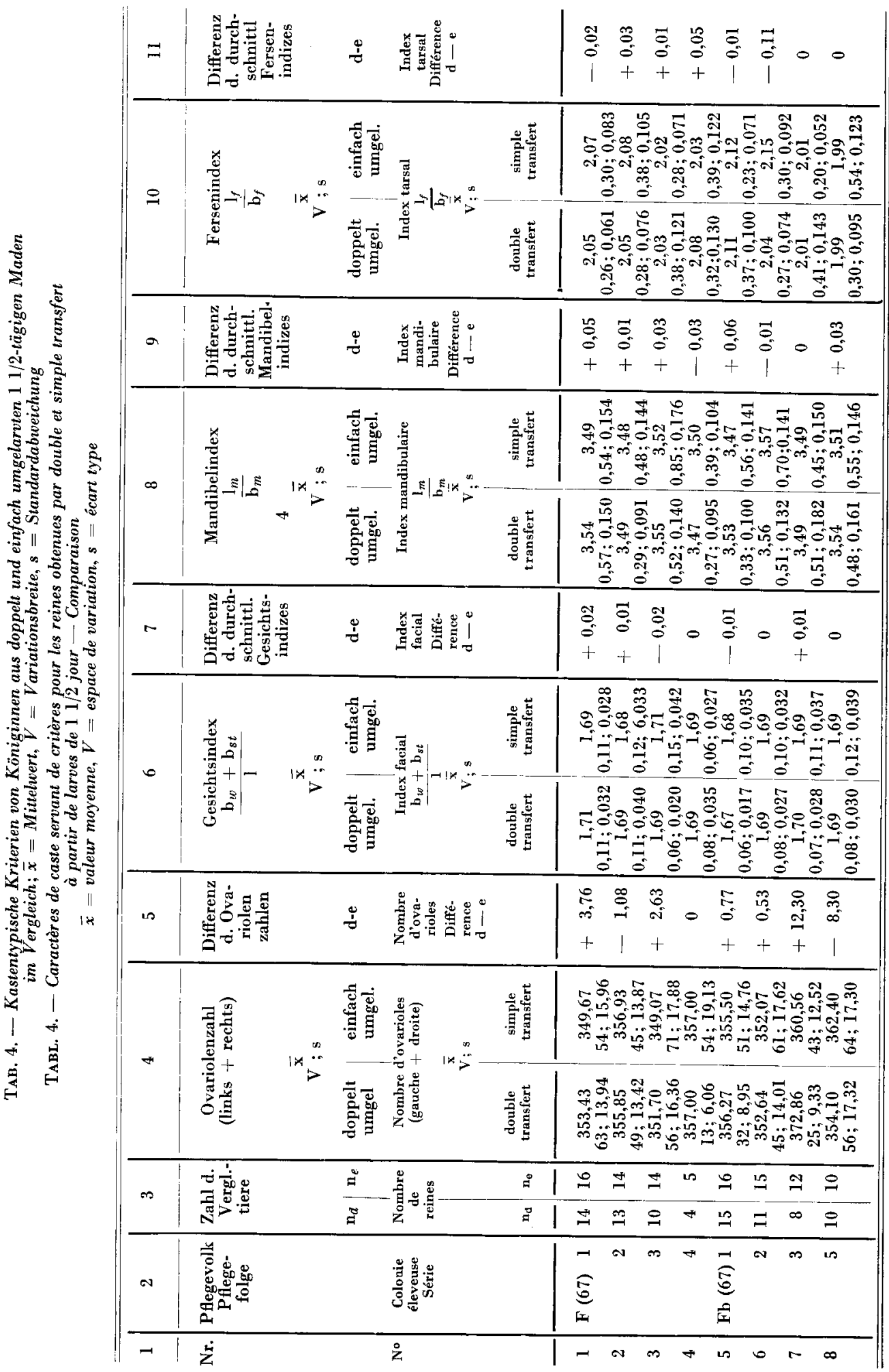


Königinnen so wenig beeinflußt hat, ist es nicht sehr wahrscheinlich, daß analytisch garnicht konstatierbare Qualitätsunterschiede im Königinnenfuttersaft von geringfügig verschieden alten Larven von Bedeutung sein können. Es ist aber nicht ausgeschlossen, daß die Menge des Futtersaftvorrates eine Rolle spielt. Beim doppelten Umlarven bestimmtalter Maden auf den Futtersaft von vortags im gleichen Alter umgebetteten Tieren befindet sich, gemessen am Alter und der Größe der Zweitlarven, zuviel Futtersaft in den Näpfchen. Die Menge des in einer Zeiteinheit in ein trockenes Näpfchen abgelagerten Futtersaftes richtet sich nach dem Umbettungsalter der Larven und ist beispielsweise 24. Stunden nach dem Umlarven 1-tägiger Maden nahezu doppelt so groß wie bei der Verwendung eben geschlüpfter Tiere (WEIss 1974). Die Folge könnte sein, daß sich die Ammenbienen um die zu reichlich vorversorgten Maden in der ersten Zeit zu wenig kümmern. Das Futter dickt ein, es kommt zu Aufnahmeschwierigkeiten und letztlich zu einer Wachstumsminderung der doppelt umgelarvten Tiere. Dagegen würde eine Made, welche ihrem Alter und ihrer Größe nach zu dem im Näpfchen befindlichen Futtersaft paßt, regulär weitergefüttert werden. Das könnte für sie gegenüber der trocken umgelarvten Made, die je nach Beschaffenheit des Pflegevolkes kürzer oder länger auf ihr erstes Futter warten muß, von Vorteil sein.

Von einem solchen Wachstumsvorteil ist allerdings beim doppelten Umlarven von 1-tägigen Maden auf altersangepaßten Futtersaft nicht viel zu merken (2. Versuchsgruppe, Tab. 3). Er wird erst bei der vergleichsweisen einfachen und doppelten Umbettung von $11 / 2$ bis 2-tägigen Maden deutlich, wobei es sogar nichts mehr ausmacht, ob das Futterpolster altersmäBig dem Entwicklungsstadium der Larven entspricht oder nicht (3. u. 4. Versuchsgruppe, Tab. 3). Offenbar sind ältere Maden in ihrem Wachstum stärker auf eine kontinuierliche Fütterung angewiesen als jüngere Tiere, welche normaerweise weniger Futter verbrauchen und auch erhalten.

Überblickt man die Schwankungen der durchschnittlichen Königinnengewichte während der einzelnen aufeinanderfolgenden Zuchtserien im jeweils gleichen Pflegevolk, so läßt sich nur sehr bedingt eine Gewichtsabnahme der Königinnen mit fortschreitendem Alter der Pflegebienen feststellen. Es gibt fast ebenso viele Ausnahmen von dieser Regel. Dagegen verringern sich die Futtersaftrestmengen, welche eine gesetzmäßige Beziehung zum doppelten und einfachen Umlarven aufweisen, mit fortschreitender Zuchtfolge sehr deutlich (Tab. 2). Schwankungen der durchschnittlichen Königinnengewichte und der Futtersaftrestversorgung in den einzelnen Zuchtserien sind manchmal, aber nicht immer, mit dem wechselnden Umfang von Larvenangebot und Annahme zu erklären. Manche Serien scheinen einfach besser gepflegt zu werden als andere. Der Grund ist unbekannt. - Ich hätte erwartet, daß sich in späteren Serien der Wiederholungszuchten bei nachlassender Pflegekraft ein Gewichtsvorteil der doppelt umgelarvten Königinnen nach- 
weisen ließe. Er trat nicht ein. Auch hier reagierten die Völker scheinbar willkürlich.

Besondere Verhältnisse herrschen, wenn (in meinen Versuchen ausnahmsweise!) die Herkunft des Zuchtstoffes innerhalb einer Pflegefolge wechselt. Dann ist auch noch mit einer genetischen Beein flussung der Gewichtsentwicklung der Königinnen zu rechnen. Tabelle 3 scheint in Zucht $\mathrm{Nr} .7 \mathrm{u}$. 15 Hinweise hierfür zu enthalten.

Bei den Zuchtversuchen mit doppelt und einfach umgelarvten Maden hatten die Bienen stets die Wahl zwischen beiden. Wenn sie die doppelt umgebetteten Larven bevorzugten, heißt das nicht, daß sie diese bei ausschließlicher Darbietung ebenfalls besser angenommen hätten. Der Annahmeprozentsatz in der Hauptzucht mit den anteilweise doppelt umgelarvten Maden ist zwar ebenfalls höher als in der Anbrütezucht mit ausschließlich einfach umgelarvten Tieren, aber das Larvenangebot war in den Hauptzuchten in der Regel auch viel kleiner (Tab. 1). Die absoluten Annahmezahlen in der Hauptzucht sind dagegen eher etwas geringer als in der Anbrütezucht. Das doppelte Umlarven hat also nicht zur Verbesserung der Annahme beigetragen. Wenn man daraus mit Vorbehalt den Schluß ziehen möchte, daß doppeltes, oder im weiteren Sinne, “ feuchtes » Umlarven bei fehlender Auswahlmöglichkeit gegenüber dem einfachen und « trockenem » Umlarven keine Annahmevorteile mit sich bringt, so geschieht das nicht ohne Einschränkung. Nach BötTcher und Werss (1962) scheinen schwache Pflegevölker Larven auf Futtersaft bevorzugt anzunehmen. Auch im “ weiselrichtigen ) Pflegevolk liegen besondere Verhältnisse vor. Dieses Verfahren, bei welchem die Aufzucht der Weiselzellen in einem von der Königin durch Absperrgitter getrennten Raum vorgenommen wird, scheint nach meiner bisherigen Erfahrung besser zu funktionieren, wenn man die Larven statt in trockene Zellen auf etwas Futtersaft umbettet. Möglicherweise wirkt die Vorversorgung der Weiselnäpfchen mit Königinnenfuttersaft der verminderten Annahmebereitschaft des weiselrichtigen Pflegevolkes entgegen. Weiselrichtige Völker nehmen auch angebrütete Näpfchen gegenüber neu belarvten bevorzugt in Pflege. Das weltweite Zuchtverfahren der weisellosen Anpflege (starter) und weiselrichtigen Endpflege (finisher) gründet sich auf diese Erfahrung. Infolge der Ähnlichkeit, welche der mit Futtersaft versorgte Weiselbecher mit einem angebrüteten Näpfchen besitzt, läßt sich die relativ bessere Annahme feucht umgebetteter Maden gegenüber trocken umgelarvten Tieren im weiselrichtigen Pflegevolk unschwer erklären.

Für die Praxis, welche nach der Zweckmäßigkeit des doppelten Umlarvens fragt, möchte ich aus dem vorliegenden Untersuchungsmaterial folgern, daß mit dem Mehraufwand dieses Verfahrens so gut wie nichts gewonnen wird. Es ist für die Größenentwicklung der Königinnen eher nach- als vorteilig, wenn man junge Maden im Alter von einem Tag auf die verhältnis- 
mäßig große Futtersaftmenge von 2-tägigen Larven bettet, d. h. also, wenn in oft geübter Weise sowohl zur Erst- als auch zur Zweitbelarvung 1-tägige Maden verwendet werden. Aber auch wenn man die l-tägigen Zuchtmaden auf ein ihrem Alter entsprechendes Futtersaftpolster legt, d. h. wenn man zuerst (recht mühsam!) eben geschlüpfte Maden umbettet, ist ein praktisch bedeutsamer Gewichtsvorteil nicht zu erwarten. Erst bei der Verwendung älterer Maden zum doppelten Umlarven - in meinen Versuchen waren sie zwischen 1 1/2 und 2 Tage alt - scheint der Gewichtsunterschied kritisch zu werden, wenn auch ein Unterschied in den übrigen Kastenmerkmalen nicht bestand (Tab. 4). Für den Praktiker ist es indessen ein Leichtes, so alte Maden von der Königinnenzucht auszuschließen. Erfahrungsgemäß ist das Umbetten 1-tägiger Maden besonders leicht. Ihr Alter bietet optimale Entwicklungsbedingungen auch bei einfacher Umbettung.

Eingegangen im November 1973. Reçu pour publication en novembre 1973.

\section{RÉSUMÉ}

Parmi les techniques courantes d'élevage des reines, celle du transfert des larves d'ouvrières dans des cupules pré-formées se place en première position. Elle consiste à déposer les jeunes larves, soit dans des cupules sèches, soit sur une couche de gelée royale. Cette seconde façon d'opérer a pour objectif d'éviter autant que possible une interruption dans l'alimentation de la larve, ce qui n'est pas le cas dans la première. Les nourrices peuvent, en effet, prendre en charge les larves transférées à sec aussi bien de façon immédiate qu'avec un retard de plusieurs heures. On constate à ce sujet des différences de réactions entre les colonies éleveuses; d'autre part les larves d'une même série d'élevage peuvent attendre leur réalimentation pendant un temps très variable. Théoriquement, l'interruption de l'alimentation des larves transférées à sec pourrait être désavantageuse pour les futures reines, soit que leur poids se trouve amoindri, soit qu'elles souffrent dans la réalisation parfaite de leurs caractères féminins essentiels. A ce propos, la littérature n'apporte pas de réponse univoque, ce qui peut s'expliquer par l'insuffisance numérique des essais ou par des différences de techniques et d'interprétation des résultats.

Pour approvisionner les cupules d'élevage on utilise de la gelée royale prélevée antérieurement dans des cellules royales naturelles ou bien on pratique la méthode du double transfert. Dans ce second cas on commence par transférer les jeunes larves à sec puis on les remplace au bout d'un certain temps de prise en élevage (en pratique, le plus souvent, 24 heures) par de nouvelles larves qui sont alors déposées sur la couche de gelée royale fraîche. On peut donc opposer le double transfert au simple transfert (ou encore transfert à sec) dans lequel les cupules vides sont garnies en une seule fois et avec la larve définitive.

Dans mes expériences j'ai constamment opéré dans chaque élevage sur la même colonie éleveuse et sur le même cadre d'élevage en alternant les transferts « doubles » et « simples ». Les cupules sèches et les cupules garnies de gelée royale recevaient en même temps des larves de même origine âgées tout juste de $1,11 / 2$ ou 2 jours.

L'acceptation (Tableau 1) des larves faisant l'objet d'un double transfert était meilleure, dans la majorité des élevages, que celle des larves transférées à sec. Mais il convient de noter 
que les abeilles avaient toujours le choix entre des cellules “ double transfert " et des cellules ( simple transfert "). Il est difficile de comparer le pourcentage d'acceptations plus important obtenu au cours de l'élevage proprement dit (qui comporte en partie des larves avec double transfert) avec le pourcentage d'acceptations au cours de la période de prise en élevage des larves lesquelles, dans ce cas, sont toutes des larves transférées à sec; en effet, au cours de l'élevage proprement dit, le nombre des larves est presque toujours fortement réduit. Les chiffres absolus d'acceptation ne sont pas dans l'élevage proprement dit meilleurs que dans la prise en élevage, ce qui ne signifie pas une amélioration des acceptations par le double transfert. Avec quelques réserves, on pourrait plutôt en conclure que les colonies éleveuses, si elles n'avaient pas le choix, accepteraient aussi bien les larves transférées une fois et à sec que les larves faisant l'objet d'un double transfert.

J'ai travaillé exclusivement dans mes expériences avec des colonies éleveuses orphelines. En règle générale, les colonies pourvues d'une reine acceptent moins facilement les larves. L'expérience des éleveurs montre probablement que dans ces colonies le transfert “ humide » procure de meilleures acceptations que le transfert à sec.

Lorsque dans la même colonie éleveuse on effectue plusieurs séries d'élevages successives, on a observé, surtout dans les premières séries alors que de gros excédents de gelée royale sont disponibles, une plus forte croissance des cellules royales contenant une larve ayant fait l'objet d'un double transfert (Fig. 1). Les quantités de nourriture restant dans les cellules après la nymphose étaient nettement plus importantes pour les larves ayant subi le double transfert que pour les autres (Tableau 2).

Malgré l'augmentation de taille des cellules royales et l'abondance des restes de nourriture, les reines obtenues par double transfert des larves n'étaient pas vraiment supérieures à celles obtenues par simple transfert en ce qui concerne le poids du corps. Lorsque tout d'abord des larves tout juste âgées de 1 jour sont transférées, puis remplacées au bout de 24 heures par des larves de même âge, on obtient même assez souvent des reines plus légères que celles qui proviennent d'un simple transfert (Tableau 3, $1^{\text {er }}$ groupe d'essais). Il semble que dans ce cas la trop grande quantité de nourriture qui se trouve dans les cellules ait une action défavorable eu égard à l'âge des larves (à peine 1 jour) qui remplacent des larves âgées de 2 jours à peine. Il est possible qu'au début les larves reçoivent trop peu de nourriture fraîche, ce qui ralentirait leur croissance.

Lorsque dans les expériences du $2^{\mathrm{e}}$ groupe on a utilisé comme première larve une larve venant d'éclore que l'on a remplacée 24 heures après par une larve de 1 jour qui s'est trouvée déposée sur une quantité de gelée royale adaptée à son âge, cet effet négatif sur la croissance ne s'est plus manifesté. En rassemblant tous les essais on constate même un léger avantage de poids par rapport aux témoins avec simple transfert. Mais ce n'est que lorsqu'on a utilisé des larves de tout juste $11 / 2$ jour à 2 jours pour le second transfert (groupes d'essais 3 et 4 ) qu'une différence nette et statistiquement significative en faveur du double transfert a pu être constatée. Dans ce cas il était alors indifférent que les larves transférées secondairement soient déposées ou non sur de la gelée royale adaptée à leur âge. Comme explication de la différence de comportement des différents stades larvaires dans le simple et le double transfert on peut penser que les larves les plus jeunes qui sont moins voraces supportent mieux une interruption éventuelle d'alimentation consécutive au transfert à sec, compte tenu de leur taux de croissance, que des larves plus âgées qui ont de plus gros besoins alimentaires.

Indépendamment du poids il n'existe aucune différence entre les reines qui proviennent du double ou du simple transfert de larves de $11 / 2$ jours. Dans les deux cas des caractères de caste aussi importants que le nombre des ovarioles, la forme de la tête, la denticulation des mandibules et les tarses de la $3^{e}$ paire de pattes sont développés de la même façon dans le sens de reines typiques (Tableau 4).

La supposition, facile à concevoir, selon laquelle l'âge de la colonie éleveuse pourrait exercer une in fluence sur la croissance pondérale relative des reines ayant subi ou non le double 
transfert, du fait que le double transfert pourrait devenir plus efficace à mesure que les réserves de gelée royale des nourrices s'amenuisent, n'a trouvé aucune confirmation dans les nombreuses séries successives d'élevages qui ont été conduites.

Pour la pratique de l'élevage des reines, je voudrais tirer les conclusions suivantes des expériences comparatives sur le simple et le double transfert :

Lorsqu'on utilise des larves jusqu'à l'âge d'un jour, le double transfert n'apporte aucun avantage convainquant. Au contraire, une couche de gelée royale trop importante par rapport à l'âge de la larve peut présenter des inconvénients en ce qui concerne le poids qui peut être atteint par l'adulte. Même si les reines de double transfert provenant de larves de 1 1/2 à 2 jours sont en règle générale plus lourdes que celles qui résultent d'un simple transfert, il n'y a là rien qui doive inquiéter l'éleveur de reines. On peut fort bien renoncer à transférer des larves aussi âgées, d'autant plus que les larves plus jeunes, et tout particulièrement celles de 1 jour, sont techniquement plus faciles à manipuler.

\section{LITERATUR}

Böttcher F.K. u. Weiss K., 1962. Zur Frage der Darbietung des Zuchtstoffs in Form von Maden. Z. Bienenf., 6 (I) 1-8.

Doolittle C. M., 1889. Scientific Queen rearing. Am. Bee J. Hamilton 1915, 1. Au flage.

Farrar C. L., 1968. Productive management of honey-bee colonies VII. Am. Bee J., 108(9) 354-356.

Free J. B. u. Spencer-Boot Yvette, 1961. Analysis of honey farmers' records on queen rearing and queen introduction. J. Acric. Sci., 56, 325-331.

Hastings J. E., 1967. Production of quality queens. Glean. Bee Cult., 95 (4) 212-219.

JoRDaN R., 1956. Das " zweimalige Umlarven », eine zweifelhafte Maßnahme! Bienenvater, 77 (6) $197-200$.

Laidlaw H. H. u. Eckert J. E., 1962. Queen Rearing. Univ. Calif. Press, Berkeley u. Los Angeles.

MăRZA E., 1965. The quality of queens obtained using different methods of preparing biological material (rum.). Lucr.stiint. Stat.cent.Seri. Apic., 6, 15-21, AA $623 / 70$.

Montagner H., 1962. Influence de la technique du double greffage sur le développement des reines de Apis mellifica. Ins. soc., IX (1), 91-99.

Örösi PÁl Z., 1952. Experiments on queen rearing I (ungar.). Allat. Kut. Evk., 1950, Budapest, 203-226 (1952). Übers. : M. A. Alber : Bee Res. Assoc. Ivo E 802.

Örösı PÁL Z., 1960. Experiments on queen rearing II (ungar.). Kis. Közl., (1) 31-79. Übers. : M. A. Alber : Bee Res. Assoc. No E 803.

ÖnösI PÁL Z., 1963. Versuche mit doppeltem Umlarven (ungar.). Méhészet, Budapest 11 (5) 83-85 AA $331 / 65$.

ÖнӧsI PÁL Z., 1966. Eiumbettung - praktisch und wissenschaftlich. Bienenvater, 87 (10) 281-299.

Pechaczex H., 1928. Massenerzeugung von Wahlköniginnen. 66. Wanderversammlung der Bienenwirte deutsch. Zunge Köln 1928. K. Wachholtz, Neumünster.

Snelgrove L. E., 1966. Queen rearing. 3. Auflage, Snelgrove, Bleadon, Sommerset.

Vuillaume, 1959. Nouvelles données sur la psychophysiologie de l'élevage des reines chez Apis mellifica. Ann. Abeille, 2 (2), 113-138.

WankLer W., 1924. Die Königin. W. Wankler, 4. Auflage, Theodor Fischer, Freiburg 1.Auflage 1903 ?

Weaver N., 1957. Experiments on dimorphism in the female honeybee. J. econ. Ent., 50 (6), $759-761$.

WEIss K., 1967. Zur vergleichenden Gewichtsbestimmung von Bienenköniginnen. Z. Bienenf. 9 (1) $1-21$.

WEISS K., 1971. Über die Ausbildung und Leistung von Königinnen aus Eiern und jungen Arbeitermaden. Apidologie 2 (1) 3-47.

WeIss K., 1974. Zur Frage des Königinnengewichtes in Abhängigkeit von Umlarvalter und Larvenversorgung. Apidologie 5 (2), 127-147.

Wohlgemuth E., 1933. Doppeltes Umlarven. Festschrift. 60.Geburtstag Prof. Zander, Liedloff, Loth u. Michaelis, Leipzig.

Wolosiewitsch A P., 1954. Vergleichende Bewertung der Methoden der künstlichen Königinnenzucht. Übers. Ber. : H. Maul, Hess. Biene, 90 (12) 352-353.

Zander E., 1953. Die Zucht der Biene. Eugen Ulmer, Stuttgart.

Zander E. u. Bötтcher F.K., 1971. Haltung und Zucht der Biene. Eugen Ulmer, Stuttgart. 